\title{
Níveis de Burnout e Bem-Estar de Estudantes de Medicina: um Estudo Transversal
}

\section{Burnout and Well-Being Levels of Medical Students: a Cross- Sectional Study}

\author{
Priscila Gadelha Cazolari ${ }^{I}$ (iD \\ Matheus de Sousa Cavalcante ${ }^{I}$ (i) \\ Marcelo Marcos Piva Demarzo ${ }^{I}\left({ }_{1}\right.$ \\ Frederico Molina Cohrs ${ }^{I}$ (D) \\ Adriana Sanudo ${ }^{I}$ (DD \\ Mariana Cabral Schveitzer ${ }^{I}$ (D)
}

\section{PALAVRAS-CHAVE}

- Qualidade de Vida.

- Estudantes de Medicina.

- Saúde Mental.

- Currículo. 


\section{KEYWORDS}

- Quality of Life.

- Medical Students.

- Mental Health.

- Curriculum.

Recebido em 14/7/19

Aceito em 10/8/20

\section{INTRODUÇÃO}

O curso de Medicina é oneroso e difícil, pois envolve várias etapas: preparação para o ingresso, processo de seleção concorrido, duração e conclusão. Ao ingressarem, é muito comum que os estudantes de Medicina apresentem também uma grande carga de estresse em meio a uma enorme gama de informações e cobranças, além de competitividade, contato com pacientes que enfrentam doenças graves, convívio com a morte, privação de lazer etc. Estudos mostram que o estresse ao qual os estudantes de Medicina estão submetidos pode aumentar ao longo do curso $^{1,2}$.

A presença de estresse crônico, causado pelas atividades e exigências do curso, pode levar ao burnout, uma síndrome caracterizada por três dimensões: exaustão emocional, desumanização (ou despersonalização) e reduzida realização profissional. A exaustão emocional se caracteriza pelo esgotamento mental e físico e pela sensação de incapacidade, que podem levar a sintomas de ansiedade e depressão. Tal condição tem como consequência (e também como causa) a desumanização, que seria um estado em que o indivíduo se torna indiferente, impessoal, irônico e cínico em relação às outras pessoas, como uma forma de distanciamento social e na tentativa de minimizar a exaustão. Finalmente, as atividades exercidas perdem o sentido e o indivíduo tem a sensação de fracasso e insatisfação ${ }^{2,3}$.

O burnout pode ter implicações na vida pessoal, como abuso de substâncias e suicídio, e nos âmbitos profissional e coletivo, o que pode resultar em risco aos próprios pacientes. Entre as principais consequências físicas do burnout, estão cansaço excessivo, distúrbios do sono, dores musculares, cefaleias, distúrbios gastrointestinais, transtornos alimentares e imunodeficiências. Os sinais e sintomas de caráter cognitivo podem incluir dificuldade de concentração, diminuição da memória e lentidão de pensamento; os de caráter emocional são irritação, ansiedade, depressão,

\section{ABSTRACT}

Introduction: The presence of chronic stress, caused by the activities and demands of the medical course, can lead to what is called 'burnout', a syndrome characterized by three dimensions: emotional exhaustion, dehumanization (or depersonalization) and reduced professional achievement. Considering the increased incidence of burnout syndrome, anxiety and depression symptoms, suicide attempts and suicide rates among medical students, as a consequence of increasing demands for professional and financial success at the expense of physical and mental health, one understands the importance of studying the subject and proposing measures of prevention and control. The aim of this study was to evaluate and describe the levels of burnout and well-being of medical students at a Brazilian public university. Method: The stress and well-being levels of students from the first to the sixth year for the Medical Course at Universidade Federal de São Paulo were evaluated using MBI-SS and WHOQOL-BREF questionnaires, applied online on the REDCAP platform. Results: A total of 302 students completed the questionnaires. Regarding the MBI-SS, students showed a low value in the emotional from low to moderate. It was also observed that female students showed a greater tendency toward emotional exhaustion when compared to male ones, as well as the fact that students from the $1^{\text {st }}$ and $2^{\text {nd }}$ years showed higher values of professional effectiveness when compared with $3^{\text {rd }}$ and $4^{\text {th }}$ years, with no difference between genders. Based on the answers from WHOQUOL-BREF questionnaire, the students thought they had a good quality of life. Discussion: These results reinforce possible factors that might interfere with the students' quality of life: excessive workload, teaching model based on extensive lectures, lack of stimulation, recognition for their efforts. Conclusion: The medical students evaluated in this study have a good quality of life and show low or moderate burnout levels. 
vivido pelos estudantes do primeiro ano. Entretanto, um terço dos estudantes teve pontuações baixas em pelo menos três dos domínios avaliados e um quarto não estava satisfeito com a saúde ${ }^{11}$.

Portanto, diante do aumento da incidência de síndrome de burnout, sintomas de ansiedade e depressão, tentativas de suicídio e suicídio entre estudantes de Medicina, o que pode ser encarado como resultado de exigências cada vez maiores de alcance de sucesso profissional e financeiro em detrimento da saúde física e mental, entende-se a importância de estudar o assunto e propor medidas de prevenção e controle.

Nesse contexto, o presente estudo teve como objetivos avaliar e descrever os níveis de burnout e bem-estar de estudantes de Medicina da Universidade Federal de São Paulo (Unifesp).

\section{MÉTODO}

O estudo é quantitativo, de caráter transversal e descritivo. Foram avaliados os níveis de estresse e qualidade de vida dos estudantes do primeiro ao sexto ano do curso de Medicina da Unifesp. O critério de inclusão era estar devidamente matriculado no curso de Medicina da Escola Paulista de Medicina da Unifesp.

A coleta de dados foi feita a partir de questionários autoinformados on-line, por meio da plataforma Research Electronic Data Capute (REDCap). Aplicaram-se os questionários Maslach Burnout Inventory - Student Survey (MBI-SS) e World Health Organization Quality of Life abreviado (Whoqol-Bref), além de um questionário sociodemográfico composto pelos seguintes dados: sexo, idade, estado civil, tipo de escola (público, privada ou misto), moradia (amigos, família, sozinho, outros) e financiamento dos estudos (bolsa, família, próprio).

O MBI-SS foi projetado para avaliar síndrome de burnout em estudantes $^{12}$ e é composto por 15 itens distribuídos entre as seguintes dimensões: exaustão (cinco perguntas), descrença (quatro perguntas) e eficácia profissional (seis perguntas). Na sua versão original, o MBI-SS permite calcular, pela soma dos itens respectivos, os escores de exaustão, descrença e eficácia profissional. Um indivíduo é diagnosticado com a síndrome de burnout, relativamente ao seu grupo, se simultaneamente estiver acima do percentil 66 dos escores de exaustão e descrença e abaixo do percentil 33 dos escores de realização ${ }^{12,13}$. Cada domínio é classificado em baixo, médio ou alto, com seus respectivos pontos de corte: exaustão emocional (baixo: menor que 19; médio: entre 19 e 26; e alto: superior ou igual a 27); descrença (baixo: menor que 6; médio: entre 6 e 9; e alto: superior ou igual a 10) e eficácia profissional (baixo: inferior ou igual a 40; médio: entre 34 e 39; e alto: inferior ou igual a 33).

O Whoqol-Bref contém 26 questões, sendo duas delas mais gerais, sobre qualidade de vida e satisfação com a saúde, e as 24 restantes são divididas em quatro domínios: físico (sete questões sobre dor e desconforto, energia e fadiga, sono e repouso, mobilidade, atividades da vida cotidiana, uso de medicamentos e capacidade para o trabalho), psicológico (seis questões sobre sentimentos positivos e negativos, pensar e aprender, memória e concentração, imagem corporal e espiritualidade), relações sociais (três questões sobre relações pessoais, suporte social e atividade sexual) e meio ambiente (oito questões sobre segurança física e proteção, ambiente do lar, recursos financeiros, disponibilidade e qualidade de cuidados de saúde e sociais, oportunidades de adquirir novas informações e habilidades, atividades de lazer, ambiente físico e transporte). A pontuação final pode ir de 0 a 100, sendo o 100 mais favorável à qualidade de vida ${ }^{14,15}$.

Um total de 720 estudantes de Medicina (120 estudantes por ano de curso - número total de vagas do primeiro ao sexto ano) foi convidado para responder aos questionários por meio de grupos de mensagem/rede social. Os questionários foram disponibilizados no período de agosto a outubro de 2017 por meio de link on-line e divulgados em redes sociais e grupos de mensagem de cada ano do curso. Submeteu-se o trabalho ao Comitê de Ética em Pesquisa e à Câmara de Graduação da Unifesp (Aprovação $\mathrm{n}^{\circ}$ 2.351.354). Todos os participantes assinaram o Termo de Consentimento Livre e Esclarecido, o qual apresentava as informações sobre a pesquisa e também sobre um meio de suporte existente na universidade para que o procurassem caso sentissem necessidade.

\section{Análise estatística}

Os dados demográficos foram descritos por meio do número e da porcentagem para as variáveis categóricas. Construíram-se gráficos do tipo boxplot para as variáveis numéricas como uma forma de avaliar a distribuição das variáveis avaliadas. A suposição de normalidade das variáveis quantitativas foi realizada por meio do teste Shapiro-Wilk. Como os dados mostraram-se normalmente distribuídos, optou-se por resumir a informação como média \pm desvio padrão.

Comparou-se o escore médio de cada dimensão do MBI-SS e do Whoqol-Bref entre os anos do curso ajustado pelo sexo por meio de uma análise de variância com dois fatores, pelo teste two-way ANOVA, e o post hoc foi realizado por meio de teste de comparações múltiplas de Bonferroni.

A correlação entre os domínios do Whoqol-Bref com as dimensões do MBI-SS foi avaliada por meio do cálculo do coeficiente de correlação de Pearson.

Em toda análise estatística, adotou-se um nível de significância de $5 \%$, ou seja, consideraram-se como estatisticamente significantes os resultados que apresentaram valor de $\mathrm{p}$ menor do que $5 \%(\mathrm{p}<0,05)$. Toda análise foi realizada através do Stata/SE 15.1 for Windows.

\section{RESULTADOS}

Dos 720 estudantes convidados para responder os questionários, obteve-se um total de 302 respostas completas para o MBI-SS e WhoqolBref $(n=302)$. A Tabela 1 apresenta os dados sociodemográficos dos estudantes incluídos.

De acordo com a Tabela 1, o sexo feminino foi o mais frequente (55\%) e a idade dos estudantes variou de 18 a 35 anos. Na maioria, esses estudantes eram solteiros (96\%), estudaram em escola privada $(67,9 \%)$ e moravam com a família (52,3\%), a qual financiava os estudos deles $(84,1 \%)$.

Com relação aos valores de corte para as dimensões do MBI-SS, no que diz respeito à exaustão emocional, dos 302 estudantes avaliados, observou-se que a maioria deles (64,9\%) teve exaustão emocional considerada baixa (<19). Para a dimensão descrença, observou-se que $86 \%$ deles apresentaram descrença elevada. Sobre eficácia profissional, todos os 302 estudantes obtiveram valores elevados, o que é considerado boa eficácia profissional. Esses resultados podem ser observados no Gráfico 1.

A Tabela 2 apresenta as médias do questionário MBI-SS dos estudantes de Medicina de acordo com sexo e ano do curso.

Sobre exaustão emocional, todos os anos avaliados (primeiro e segundo, terceiro e quarto, quinto e sexto) obtiveram valores baixos para exaustão (média < 19), e não houve diferença estatisticamente significante

REVISTA BRASILEIRA DE EDUCAÇÃO MÉDICA

3 44 (4) : e125; 2020 


\begin{tabular}{|c|c|}
\hline \multicolumn{2}{|c|}{ Tabela 1} \\
\hline \multicolumn{2}{|c|}{ Descrição dos 302 estudantes participantes da pesquisa. São Paulo, 2017} \\
\hline & No $(\%)$ \\
\hline \multicolumn{2}{|l|}{ Gênero $^{a}$} \\
\hline Feminino & $165(55,0 \%)$ \\
\hline Masculino & $135(65,0 \%)$ \\
\hline \multicolumn{2}{|l|}{ Idade, anos } \\
\hline Média \pm desvio padrão & $23,2 \pm 3,0$ \\
\hline Mínimo - máximo & $18-35$ \\
\hline \multicolumn{2}{|l|}{ Ano curso } \\
\hline $1^{\circ} \mathrm{e} 2^{\circ}$ ano & $113(37,4 \%)$ \\
\hline $3^{\circ} \mathrm{e} 4^{\circ}$ ano & $85(28,2 \%)$ \\
\hline $5^{\circ}$ e $6^{\circ}$ ano & $104(34,4 \%)$ \\
\hline \multicolumn{2}{|l|}{ Estado civil ${ }^{\mathrm{a}}$} \\
\hline Solteiro & $288(96,0 \%)$ \\
\hline União estável & $12(4,0 \%)$ \\
\hline \multicolumn{2}{|l|}{ Tipo de escola } \\
\hline Público & $61(20,2 \%)$ \\
\hline Misto & $36(11,9 \%)$ \\
\hline Privado & $205(67,9 \%)$ \\
\hline \multicolumn{2}{|l|}{ Moradia } \\
\hline Família & $158(52,3 \%)$ \\
\hline Amigos & $88(29,1 \%)$ \\
\hline Sozinho & $48(15,9 \%)$ \\
\hline Outros & $8(2,7 \%)$ \\
\hline \multicolumn{2}{|l|}{ Financiamento dos estudos } \\
\hline Família & $254(84,1 \%)$ \\
\hline Bolsa & $9(3,0 \%)$ \\
\hline Próprio & $10(3,3 \%)$ \\
\hline Família + Bolsa & $18(6,0 \%)$ \\
\hline Família + Próprio & $8(2,6 \%)$ \\
\hline Família + Bolsa + Próprio & $(1,0 \%)$ \\
\hline
\end{tabular}

${ }^{2}$ Dois questionários sem informação. Fonte: Elaborada pelos autores.

( $p>0,05)$ entre os anos avaliados.

A respeito da descrença, observou-se que todos os anos avaliados apresentaram altos valores para descrença (média $\geq 10$ ), sem haver diferença significante entre os anos do curso.

Para eficácia profissional, observou-se que todos os anos apresentaram valores médios baixos, o que inversamente indica boa eficácia profissional. Contudo, houve diferença estatisticamente significante; os primeiro e segundo anos apresentaram média de eficácia profissional maior do que a apresentada pelos terceiros e quarto anos $(\mathrm{p}=0,009)$.

Em relação ao sexo, os dados sugerem uma leve tendência de maior exaustão entre as mulheres no terceiro e quarto ano. Não houve diferença

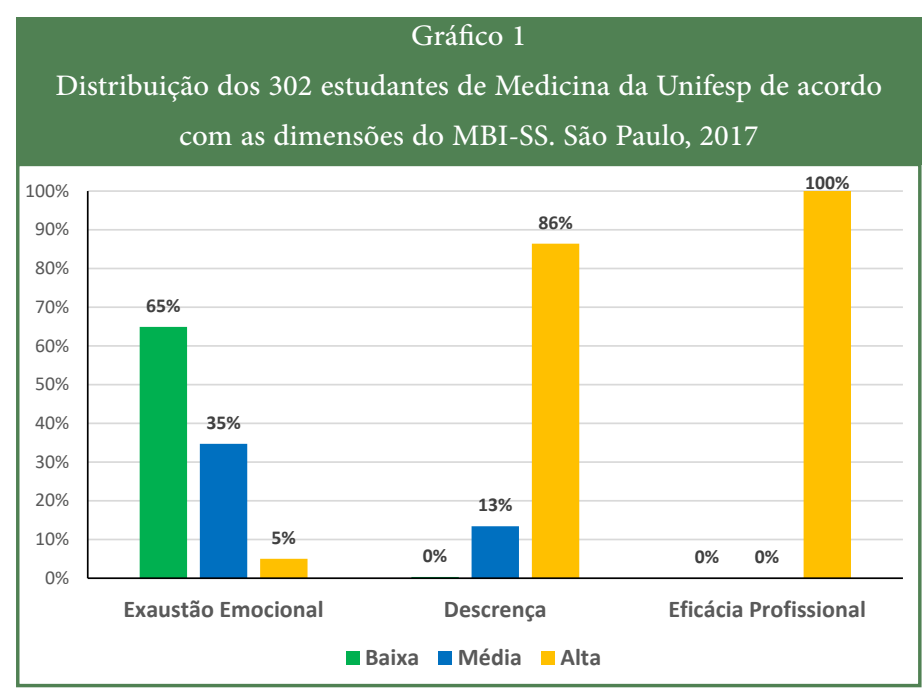

Fonte: Elaborado pelos autores.

\begin{tabular}{|c|c|c|c|c|c|}
\hline \multicolumn{6}{|c|}{ Tabela ? } \\
\hline \multicolumn{6}{|c|}{$\begin{array}{l}\text { Média } \pm \text { desvio padrão dos valores de exaustão emocional, } \\
\text { descrença e eficácia profissional, de acordo com ano do curso e sexo } \\
\text { do questionário MBI-SS dos estudantes de Medicina da Unifesp, } \\
\text { São Paulo, } 2017\end{array}$} \\
\hline \multirow{2}{*}{ MBI-SS } & \multicolumn{5}{|c|}{ Anos do curso } \\
\hline & & $1^{\circ}$ e $2^{\circ}$ & $3^{\circ}$ e $4^{\circ}$ & $5^{\circ}$ e $6^{\circ}$ & p-valor \\
\hline \multirow{3}{*}{$\begin{array}{c}\text { Exaustão } \\
\text { emocional }\end{array}$} & Feminino & $\begin{array}{c}17,8 \pm 3,5 \\
(\mathrm{n}=58)\end{array}$ & $\begin{array}{c}17,3 \pm 3,4 \\
(\mathrm{n}=45)\end{array}$ & $\begin{array}{c}17,1 \pm 3,4 \\
(\mathrm{n}=62)\end{array}$ & 0,538 \\
\hline & Masculino & $\begin{array}{c}17,1 \pm 3,9 \\
(\mathrm{n}=55)\end{array}$ & $\begin{array}{c}15,6 \pm 3,1 \\
(\mathrm{n}=39)\end{array}$ & $\begin{array}{c}15,7 \pm 3,7 \\
(\mathrm{n}=41)\end{array}$ & \multirow[t]{2}{*}{0,054} \\
\hline & p-valor & 0,330 & 0,003 & 0,004 & \\
\hline \multirow{3}{*}{ Descrença } & Feminino & $\begin{array}{c}13,3 \pm 4,1 \\
(\mathrm{n}=58)\end{array}$ & $\begin{array}{c}14,5 \pm 4,3 \\
(\mathrm{n}=45)\end{array}$ & $\begin{array}{c}15,6 \pm 4,3 \\
(\mathrm{n}=62)\end{array}$ & 0,009 \\
\hline & Masculino & $\begin{array}{c}14,1 \pm 3,4 \\
(\mathrm{n}=55)\end{array}$ & $\begin{array}{c}13,2 \pm 3,8 \\
(\mathrm{n}=39)\end{array}$ & $\begin{array}{c}13,8 \pm 3,8 \\
(\mathrm{n}=41)\end{array}$ & \multirow[t]{2}{*}{0,528} \\
\hline & p-valor & 0,285 & 0,865 & 0,591 & \\
\hline \multirow{3}{*}{$\begin{array}{c}\text { Eficácia } \\
\text { profissional }\end{array}$} & Feminino & $\begin{array}{c}19,7 \pm 4,1 \\
(\mathrm{n}=58)\end{array}$ & $\begin{array}{c}18,6 \pm 4,3 \\
(\mathrm{n}=45)\end{array}$ & $\begin{array}{c}18,7 \pm 4,3 \\
(\mathrm{n}=62)\end{array}$ & 0,284 \\
\hline & Masculino & $\begin{array}{c}19,3 \pm 3,4 \\
(\mathrm{n}=55)\end{array}$ & $\begin{array}{c}16,6 \pm 3,8 \\
(\mathrm{n}=39)\end{array}$ & $\begin{array}{c}17,7 \pm 3,8 \\
(\mathrm{n}=41)\end{array}$ & \multirow[t]{2}{*}{0,006} \\
\hline & p-valor & 0,551 & $<0,001$ & 0,013 & \\
\hline
\end{tabular}

Fonte: Elaborada pelos autores.

em relação à descrença, e, quanto à eficácia profissional, os dados indicam maiores valores entre as mulheres.

Em relação à qualidade de vida dos estudantes a partir das respostas do Whoqol-Bref, os dados foram analisados a partir de cada domínio e organizados no Gráfico 2. As médias de cada escore foram as seguintes: meio ambiente $(70,4)$, relações sociais $(68,7)$, psicológico $(65,2)$ e físico (67.9), consideradas entre regular e boa, sem diferença estatística entre os domínios avaliados, tanto entre os sexos quanto entre os anos de curso (Tabela 3). 


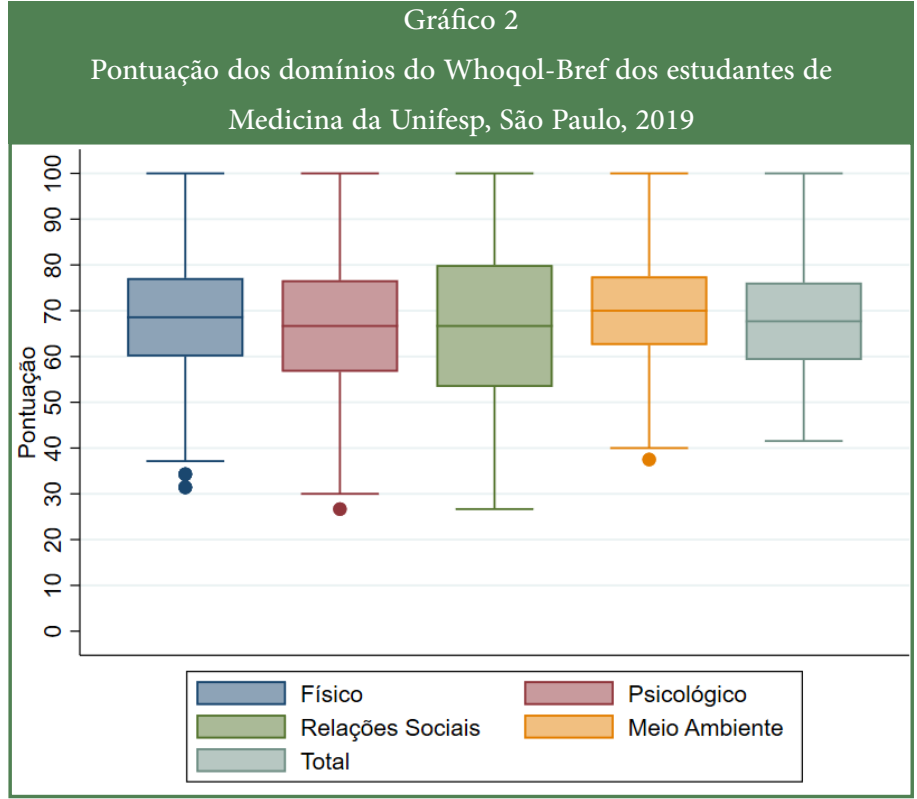

Fonte: Elaborado pelos autores.

\section{Tabela 3}

Média \pm desvio padrão dos domínios do Whoqol dos estudantes

de Medicina da Unifesp, de acordo com ano do curso e sexo, São Paulo, 2019

\begin{tabular}{|c|c|c|c|c|c|}
\hline \multirow{2}{*}{ Whoqol } & \multicolumn{5}{|c|}{ Anos do curso } \\
\hline & & $1^{\circ}$ e $2^{\circ}$ & $3^{\circ} \mathrm{e} 4^{\circ}$ & $5^{\circ} \mathrm{e} 6^{\circ}$ & p-valor \\
\hline \multirow{3}{*}{$\begin{array}{c}\text { Domínio } \\
\text { Físico }\end{array}$} & Feminino & $\begin{array}{c}14,1 \pm 2,5 \\
(\mathrm{n}=93)\end{array}$ & $\begin{array}{c}12,9 \pm 2,6 \\
(\mathrm{n}=65)\end{array}$ & $\begin{array}{c}12,5 \pm 2,5 \\
(\mathrm{n}=89)\end{array}$ & 0,004 \\
\hline & Masculino & $\begin{array}{c}14,6 \pm 2,3 \\
(\mathrm{n}=55)\end{array}$ & $\begin{array}{c}14,2 \pm 2,9 \\
(\mathrm{n}=66)\end{array}$ & $\begin{array}{c}14,0 \pm 2,7 \\
(\mathrm{n}=53)\end{array}$ & 0,596 \\
\hline & p-valor & 0,729 & 0,751 & 0,540 & \\
\hline \multirow{3}{*}{ Psicológico } & Feminino & $\begin{array}{c}13,4 \pm 2,6 \\
(\mathrm{n}=93)\end{array}$ & $\begin{array}{c}12,3 \pm 2,8 \\
(\mathrm{n}=65)\end{array}$ & $\begin{array}{c}12,0 \pm 2,9 \\
(\mathrm{n}=89)\end{array}$ & 0,023 \\
\hline & Masculino & $\begin{array}{c}13,5 \pm 2,5 \\
(\mathrm{n}=55)\end{array}$ & $\begin{array}{c}14,0 \pm 3,3 \\
(\mathrm{n}=66)\end{array}$ & $\begin{array}{c}13,4 \pm 3,1 \\
(\mathrm{n}=53)\end{array}$ & 0,448 \\
\hline & p-valor & 0,969 & 0,251 & 0,983 & \\
\hline \multirow{3}{*}{$\begin{array}{c}\text { Relações } \\
\text { sociais }\end{array}$} & Feminino & $\begin{array}{c}13,8 \pm 3,1 \\
(\mathrm{n}=93)\end{array}$ & $\begin{array}{c}13,2 \pm 3,2 \\
(\mathrm{n}=65)\end{array}$ & $\begin{array}{c}13,5 \pm 3,4 \\
(\mathrm{n}=89)\end{array}$ & 0,183 \\
\hline & Masculino & $\begin{array}{c}13,7 \pm 2,9 \\
(\mathrm{n}=55)\end{array}$ & $\begin{array}{c}14,5 \pm 3,7 \\
(\mathrm{n}=66)\end{array}$ & $\begin{array}{c}13,8 \pm 3,4 \\
(n=53)\end{array}$ & 0,209 \\
\hline & p-valor & 0,885 & 0,118 & 0,948 & \\
\hline \multirow{3}{*}{$\begin{array}{c}\text { Meio } \\
\text { ambiente }\end{array}$} & Feminino & $\begin{array}{c}14,6 \pm 2,4 \\
(\mathrm{n}=93)\end{array}$ & $\begin{array}{c}13,6 \pm 2,4 \\
(\mathrm{n}=65)\end{array}$ & $\begin{array}{c}13,3 \pm 2,7 \\
(\mathrm{n}=89)\end{array}$ & 0,001 \\
\hline & Masculino & $\begin{array}{c}14,3 \pm 2,1 \\
(\mathrm{n}=55)\end{array}$ & $\begin{array}{c}15,1 \pm 2,8 \\
(\mathrm{n}=66)\end{array}$ & $\begin{array}{c}14,3 \pm 3,0 \\
(\mathrm{n}=53)\end{array}$ & 0,241 \\
\hline & p-valor & 0,395 & 0,463 & 0,379 & \\
\hline \multirow{3}{*}{ Total } & Feminino & $\begin{array}{c}14,1 \pm 2,1 \\
(\mathrm{n}=93)\end{array}$ & $\begin{array}{c}13,0 \pm 2,2 \\
(\mathrm{n}=65)\end{array}$ & $\begin{array}{c}12,7 \pm 2,2 \\
(\mathrm{n}=89)\end{array}$ & 0,002 \\
\hline & Masculino & $\begin{array}{c}14,2 \pm 1,8 \\
(\mathrm{n}=55)\end{array}$ & $\begin{array}{c}14,5 \pm 2,7 \\
(\mathrm{n}=66)\end{array}$ & $\begin{array}{c}14,0 \pm 2,5 \\
(n=53)\end{array}$ & 0,344 \\
\hline & p-valor & 0,859 & 0,304 & 0,646 & \\
\hline
\end{tabular}

Fonte: Elaborada pelos autores.
Quando se correlacionaram os dados, observou-se correlação negativa entre todos os domínios do Whoqol-Bref e as dimensões do MBI-SS. Isto é, notou-se que, ao passo que há aumento dos domínios físico, psicológico, relações sociais e meio ambiente, caracterizando boa qualidade de vida, há diminuição das dimensões de exaustão emocional, descrença e de pouca eficácia profissional. Sendo a recíproca também verdadeira. Isto é, diminuições dos domínios de qualidade de vida estão relacionados a aumento das dimensões de burnout do MBI-SS (Tabela 4).

\section{DISCUSSÃO}

Um alto nível de burnout está relacionado com a apresentação concomitante de altos escores nos fatores de exaustão e descrença e baixos escores na dimensão eficácia profissional, enquanto um nível moderado refere-se à presença de escores médios nas três subescalas e um nível baixo está relacionado à existência de baixos escores nos fatores exaustão e descrença e de altos escores no fator eficácia profissional. No presente estudo, os estudantes apresentaram baixo valor no fator exaustão emocional e altos valores na descrença e eficácia profissional, indicando um burnout entre baixo e moderado ${ }^{12,13}$.

Neste estudo, apenas foi encontrada diferença estatística na dimensão eficácia profissional na comparação entre o primeiro e segundo anos versus o terceiro e quarto anos. Com base nesses resultados, pode-se considerar que os estudantes dos primeiros anos estão menos expostos à carga estressora e a outros fatores quando comparados com os discentes de anos mais avançados. Além disso, pode haver uma perda de idealizações da carreira à medida que os estudantes se deparam com a realidade da medicina prática, o que explicita as diferenças concernentes à realização profissional ${ }^{16}$. Destacam-se a complexidade e a multiplicidade de funções e tarefas que aumentam conforme os anos do curso avançam, além da pressão para aprovação em exames e/ou avaliações educacionais e processos de seleção para residência médica, que começam a surgir e fazer parte da rotina de estudantes mais velhos, principalmente entre aqueles no quinto e sexto anos ${ }^{16}$. Tamayo ${ }^{17}$ também observou maiores níveis de burnout nos dois últimos anos, os quais correspondem ao internato.

Outro problema muito presente associado ao burnout em estudantes de Medicina refere-se aos distúrbios do sono ${ }^{18}$. Um estudo realizado em escola médica de Recife $^{19}$ mostrou que porcentagens significativas

\begin{tabular}{|cccc|}
\hline \multicolumn{4}{c}{ Tabela 4} \\
\hline $\begin{array}{c}\text { Correlação* entre os domínios do Whoqol-Bref e as dimensões do } \\
\text { MBI-SS }\end{array}$ \\
\hline $\begin{array}{c}\text { Domínios } \\
\text { Whoqol-Bref }\end{array}$ & $\begin{array}{c}\text { MBI-SS - Exaustão } \\
\text { emocional }\end{array}$ & $\begin{array}{c}\text { MBI-SS - } \\
\text { Descrença }\end{array}$ & $\begin{array}{c}\text { MBI-SS - Eficácia } \\
\text { Profissional }\end{array}$ \\
\hline Físico & $-0,439$ & $-0,565$ & $-0,313$ \\
\hline Psicológico & $-0,383$ & $-0,537$ & $-0,299$ \\
\hline Relações sociais & $-0,285$ & $-0,267$ & $-0,247$ \\
\hline Meio ambiente & $-0,249$ & $-0,248$ & $-0,148$ \\
\hline TOTAL & $-0,424$ & $-0,513$ & $-0,309$ \\
\hline
\end{tabular}

*Correlação de Pearson.

Fonte: Elaborada pelos autores. 
dos estudantes de Medicina relataram má qualidade do sono e uma porcentagem menor, porém importante, referiu a presença de possível distúrbio do sono. Além disso, o estudo também apontou que os escores relacionados ao estresse ficaram com valores acima do corte considerado para alto nível, além de qualidade de vida considerada regular e rastreio para presença de transtorno psicopatológico com porcentagem elevada de suspeita, com predomínio no sexo feminino. Pesquisar burnout e distúrbios do sono é importante porque juntos esses dois problemas podem levar a consequências, como baixo rendimento escolar, abuso de drogas lícitas e/ou ilícitas, ansiedade, depressão, ideação suicida e suicídio ${ }^{20,21}$. Todos esses fatores, que podem surgir a partir de condições impostas durante a graduação, afetam a qualidade de vida dos acadêmicos.

O nosso estudo mostrou maior tendência das mulheres à exaustão emocional no terceiro e quarto anos quando comparadas aos homens. Outros estudos também apontam importante recorte de gênero e diferença entre homens e mulheres no que diz respeito a níveis de burnout e qualidade de vida em geral, a partir dos quais as mulheres que estudam Medicina apresentam menores escores de saúde mental quando comparadas com os homens ${ }^{22}$. O que se tem explanado sobre esses resultados e essas diferenças pautados em gênero são estudos que correlacionam achados de pior escore de saúde mental e exaustão emocional com a alta prevalência de transtornos psiquiátricos, como ansiedade e depressão em estudantes mulheres, tal qual sua maior predominância quando comparadas com estudantes homens, o que refletiria os baixos valores encontrados quando se avaliam burnout e outros componentes que contemplam saúde mental ${ }^{23}$.

Outro ponto interessantemente analisado na literatura é a personalidade de aprendizado acadêmico com possíveis diferenças de gênero, o que refletiria os diferentes valores encontrados para homens e mulheres e abre margem para pesquisas futuras a respeito das metodologias de ensino que considerem perfis e outras características sociodemográficas antes de serem implementadas ${ }^{23}$. Além disso, os achados na literatura apontam os estereótipos e as disparidades de gênero fortemente presentes não só nos cursos médicos, mas também na sociedade como um todo, $\mathrm{e}$ que se relacionam com índices mais elevados de burnout e piores escores de saúde mental em geral $^{23-25}$

A partir das respostas do Whoqol-Bref, os estudantes consideraram ter boa qualidade de vida, resultado semelhante ao encontrado em Sorocaba por Ramos-Dias et al. ${ }^{11}$, porém sentem-se prejudicados na realização de suas tarefas diárias, na capacidade de trabalho e na qualidade de seu sono. Nesse estudo, não foi realizada análise comparando a qualidade de vida em cada ano do curso, e na literatura não existe consenso de como a qualidade de vida se altera com o evoluir do curso. Chazan et al. ${ }^{26}$ constataram que a qualidade de vida dos estudantes piora ao longo da graduação, e Cavalcante et al. ${ }^{27}$ compararam dados dos estudantes do primeiro e sexto anos da Unifesp e não encontraram diferença estatística em 2017, mas em 2018 verificaram menores escores no primeiro ano em comparação ao sexto.

A análise dos domínios contidos no Whoqol-Bref (físico, psicológico, relações sociais e meio ambiente) demonstrou que não há diferença estatística entre eles, havendo maior pontuação no domínio meio ambiente, seguido por relações sociais, ficando o psicológico com menor pontuação. Os valores para cada domínio foram menores do que os encontrados por Ramos-Dias et al. ${ }^{11}$ e Chazan et al. ${ }^{26}$, além de haver discordância nos valores de cada domínio. Para esses autores, os domínios físico e relações sociais foram os que mais pontuaram.

Em Ouro Preto, um estudo, no qual se utilizaram a técnica de grupo focal e o questionário do Whoqol-Bref, concluiu que as extensas cargas horárias curriculares e extracurriculares contribuem sobremaneira para a piora da qualidade de vida dos acadêmicos, pois impedem que os discentes pratiquem atividades de lazer, descansem, tenham cuidados com a saúde e adotem uma alimentação adequada ${ }^{28}$. Outro estudo realizado no Rio de Janeiro concluiu também que há uma queda na qualidade de vida dos estudantes ao longo da graduação ${ }^{26}$. Do mesmo modo, um estudo realizado na Malásia ${ }^{29}$ mostrou que condições psicopatológicas como a ansiedade e a depressão foram associadas a uma qualidade de vida pior. Os estudantes de Medicina com sintomas depressivos tiveram escores associados a menor domínio físico, psicológico e ambiental, enquanto aqueles com sintomas de ansiedade apresentaram menor escore nos domínios psicológico, social e ambiental. Ademais, esse estudo encontrou correlação negativa entre burnout e qualidade de vida, isto é, altos índices de burnout associados a baixos índices de qualidade de vida, com a recíproca também verdadeira, o que está em congruência com a literatura que analisou diversos fatores que influenciam burnout e qualidade de vida.

Alguns estudos analisam e mostram a associação entre o desenvolvimento de burnout e sintomas de ansiedade e depressão em estudantes de medicina. Dois deles revelam uma associação positiva entre um estilo de vida saudável, com prática de atividade física e alimentação equilibrada, e menores níveis de burnout nos estudantes ${ }^{30,31}$. Outro estudo, realizado no México, afirma que há uma importante relação entre um tipo de personalidade resistente e o burnout. Ele sugere que indivíduos com esse tipo de personalidade apresentam melhor capacidade de perceber situações de estresse e lidar com elas, o que seria um fator protetor para sua saúde mental e consequentemente para o estabelecimento da síndrome de burnout $^{32}$. Em contrapartida, uma revisão narrativa norteamericana afirma que as condições de estudos e trabalho, muito mais que características individuais, são as maiores determinantes do bem-estar do estudante de medicina e as causadoras de burnout ${ }^{2}$. Além disso, um estudo realizado na África do Sul mostrou que índices elevados de burnout estão associados a valores regulares e baixos nos escores de qualidade de vida avaliados pelo Whoqol-Bref principalmente no domínio psicológico ${ }^{33}, o$ que também corrobora os achados desta pesquisa.

A respeito dos resultados encontrados, podem-se indicar como limitações do estudo a amostra por conveniência e a impossibilidade de generalizar os resultados obtidos.

Finalmente, dada a relevância do assunto, a literatura aponta diferentes estratégias que podem ajudar a promover a saúde e qualidade de vida entre os estudantes, como a realização de rodas de conversa sobre compaixão $^{34}$ e cursos de mindfulness ${ }^{35}$, a fim de criar uma autoavaliação crítica dos estudantes e facilitar o acesso a medidas de ajuda, com olhar institucional para mudança de paradigmas, aproximando os discentes de mecanismos que contribuam para melhorar os aspectos abordados.

\section{CONCLUSÃO}

Com base nos resultados obtidos, é possível concluir que os estudantes de Medicina avaliados neste estudo têm boa qualidade de vida e níveis baixos ou moderados de burnout, com correlação negativa entre os achados. Há diferença significante em relação a gênero e exaustão emocional, com mulheres apresentando piores resultados do que homens, 
bem como estudantes dos primeiros anos apresentando maiores índices de realização profissional quando comparados com aqueles dos anos mais avançados. Nesse sentido, é de grande importância divulgar os resultados encontrados para os servidores da universidade e criar mecanismos institucionais para promover a saúde mental dos estudantes de Medicina da instituição, atentando-se para recortes de variáveis sociodemográficas e características específicas.

\section{AGRADECIMENTO}

Este trabalho foi realizado com auxílio de Bolsa de Iniciação à Gestão (BIG) da Pró-Reitoria de Assuntos Estudantis (Prae) da Unifesp para a primeira autora.

\section{REFERÊNCIAS}

1. Benevides-Pereira AM, Gonçalves MB. Transtornos emocionais e a formação em Medicina: um estudo longitudinal. Rev Bras Educ Med. 2009;33(1):10-23. doi: 10.1590/S0100-55022009000100003.

2. Dyrbye L, Shanafelt T. A narrative review on burnout experienced by medical students and residents. Med Educ. 2016;50(1):132-49. doi: 10.1111/medu.12927.

3. Goni M, Urgoiti, M, Durante E. Correlación entre clima educativo y síndrome de burnout em practicantes internos de la carrera de doctor em medicina de Uruguay. Rev Méd Urug. 2015;31(4):27281 [acesso em 4 dez 2019]. Disponível em: http://www.scielo.edu.uy/ scielo.php?script=sci_arttext\&pid=S1688-03902015000400007\&lng= es\&nrm=iso.

4. Almeida GC, Souza HR, Almeida PC, Almeida BC, Almeida GH. The prevalence of burnout syndrome in medical students. Arch Clin Psychiatry. 2016;43(1):6-10. doi:10.1590/0101-60830000000072.

5. Tabalipa FO, Souza MF, Pfützenreuter G, Lima VC, Traebert E. Prevalence of anxiety and depression among medical students. Rev Bras Educ Med. 2015;39(3):388-94 [acesso em 4 dez 2019]. Disponível em: http://www.scielo.br/scielo.php?script=sci_arttext\&pid=S010055022015000300388\&lng=en\&nrm=iso.

6. Baldassin S, Alves TC, de Andrade AG, Nogueira Martins LA. The characteristics of depressive symptoms in medical students during medical education and training: a cross-sectional study. BMC Med Educ. 2008;8:60 [acesso em 4 dez 2019]. Disponível em: https://www. ncbi.nlm.nih.gov/pmc/articles/PMC2621219/.

7. Pereira GA, Capanema HXM, Silva MMQ, Garcia IL, Petroianu A. Prevalência de síndromes funcionais em estudantes e residentes de Medicina. Rev Bras Educ Med. 2015;39(3):395-400 [acesso em 4 dez 2019]. Disponível em: http://www.scielo.br/pdf/rbem/v39n3/19815271-rbem-39-3-0395.pdf.

8. Vasconcelos TC, Dias BR, Andrade LR, Melo GF, Barbosa L, Souza E. Prevalência de sintomas de ansiedade e depressão em estudantes de Medicina. Rev Bras Educ Med. 2015;39(1):135-42 [acesso em 4 dez 2019]. Disponível em: http://www.scielo.br/scielo.php?script=sci arttext\&pid=S0100-55022015000100135\&lng=en\&nrm=iso.

9. Olmo NRS, Ferreira LF, Prado AD, Martins LC, Dedivitis RA. Percepção dos estudantes de Medicina do primeiro e sexto anos quanto à qualidade de vida. Diagn Tratamento. 2012;17(4):157-61.

10. Development of the World Health Organization Whoqol-Bref quality of life assessment. The Whoqol Group. Psychol Med. 1998;28(3):551-8.
11. Ramos-Dias JCR, Libardi MC, Zilo CM, Igarashi MH, Senger MH. Qualidade de vida em cem alunos do curso de Medicina de Sorocaba - PUC/SP. Rev Bras Educ Med. 2010;34(1):116-23 [acesso em 4 dez 2019]. Disponível em: http://www.scielo.br/ scielo.php?script=sci_arttext\&pid=S0100-55022010000100014\&ln $\mathrm{g}=\mathrm{en} \& \mathrm{nrm}=$ iso.

12. Carlotto MS, Câmara SG. Características psicométricas do Maslach Burnout Inventory - Student Survey (MBI-SS) em estudantes universitários brasileiros. PsicoUSF. 2006;11(2):167-73 [acesso em 4 dez 2019]. Disponível em: http://pepsic.bvsalud.org/scielo.php?script=sci_ arttext\&pid=S1413-82712006000200005\&lng=pt\&tlng=pt.

13. Maroco J, Tecedeiro M. Inventário de burnout de Maslach para estudantes portugueses. Psic Saúde \& Doenças. 2009;10(2):227-35 [acesso em 4dez2019].Disponívelem:http://www.scielo.mec.pt/scielo. php?script=sci_arttext\&pid=S1645-00862009000200007\&lng=pt.

14. Fleck MP, Leal OF, Louzada S, Xavier M, Chachamovich E, Vieira G, et al. Desenvolvimento da versão em português do instrumento de avaliação de qualidade de vida da OMS (Whoqol-100). Rev Bras Psiquiatr. 1999;21(1):19-28. doi: 10.1590/S1516-44461999000100006.

15. Fleck MP, Louzada S, Xavier M, Chachamovich E, Vieira G, Santos L, et al. Aplicação da versão em português do Instrumento Abreviado de Avaliação da Qualidade de Vida "Whoqol-bref". Rev Saúde Pública. 2000;34(2):178-83 [acesso em 4 dez 2019]. Disponível em: http://www.scielo.br/scielo.php?script=sci_arttext\&pid=S0034$89102000000200012 \& \operatorname{lng}=$ en $\& n r m=$ iso.

16. Amor EM, Baños JE, Sentí M. Prevalencia del síndrome de burnout entre los estudiantes de medicina y su relación con variables demográficas, personales y académicas. Rev Fund Educ Méd. 2020;23(1):25-33 [acesso em 26 jul 2020]. Disponível em: http://scielo.isciii.es/scielo.php?script=sci_arttext\&pid=S201498322020000100005\&lng=es.

17. Tamayo MR. Relação entre a síndrome de burnout e os valores organizacionais no pessoal de enfermagem de dois hospitais públicos [dissertação]. Brasília: Universidade de Brasília; 1997.

18. Pagnin D, de Queiroz V. Influence of burnout and sleep difficulties on the quality of life among medical students. SpringerPlus. 2015;4:676. doi: 10.1186/s40064-015-1477-6.

19. Amorim BB, Moraes L, Sá ICG, Gomes Silva BB, Camara Filho JW. Saúde mental do estudante de medicina: psicopatologia, estresse, sono e qualidade de vida. Rev Psic Divers e Saúde. 2018;7(2):245-54 [acesso em 4 dez 2019]. Disponível em: https://www5.bahiana.edu. br/index.php/psicologia/article/view/1911/1829.

20. Gómez HP, Pérez VC, Parra PP, Ortiz ML, Matus BO, McColl CP, et al. Relación entre el bienestar y el rendimiento académico en alumnos de primer año de medicina. Rev Méd Chile. 2015; 143(7):930-7. doi: 10.4067/S0034-98872015000700015.

21. Mori MO, Valente TCO, Nascimento LFC. Síndrome de burnout e rendimento acadêmico em estudantes da primeira à quarta série de um curso de graduação em Medicina. Rev Bras Educ Med. 2012;36(4):536-40 [acesso em 4 dez 2019]. Disponível em: http://www.scielo.br/scielo.php?script=sci_arttext\&pid=S0100$55022012000600013 \& \operatorname{lng}=$ en\&nrm $=$ iso.

22. Solis AC, Lotufo-Neto F. Predictors of quality of life in Brazilian medical students: a systematic review and meta-analysis. Braz J

REVISTA BRASILEIRA DE EDUCAÇÃO MÉDICA

$744(4):$ e125; 2020 
Psychiatry. 2019;41(6):556-567. doi: 10.1590/1516-4446-2018-0116.

23. Burger PHM, Scholz M. Gender as an underestimated factor in mental health of medical students. Ann Anat. 2018;218:1-6. doi: 10.1016/j.aanat.2018.02.005.

24. Jena $A B$, Khullar D, Ho O, Olenski AR, Blumenthal DM. Sex differences in academic rank in US medical schools in 2014. JAMA. 2015;314(11):1149-58. doi: 10.1001/jama.2015.10680.

25. Choo EK, van Dis J, Kass D. Time's up for medicine? Only time will tell. N Engl J Med. 2018;379(17):1592-3. doi: 10.1056/NEJMp1809351.

26. Chazan AC, Campos MR, Portugal FB. Qualidade de vida de estudantes de Medicina da Uerj por meio do Whoqol-Bref: uma abordagem multivariada. Ciênc Saúde Colet. 2015;20(2):547-56. doi: 10.1590/1413-81232015202.05182014.

27. Cavalcante M, Cazolari P, Galliano S, Cohrs F, Sañudo A, Schveitzer M. Qualidade de vida dos estudantes do primeiro e sexto ano do curso de Medicina. Rev Med (São Paulo). 2019;98(2):99-107. doi: 10.11606/issn.1679-9836.v98i2p99-107.

28. Figueiredo AM, Ribeiro GM, Reggiani ALM, Pinheiro BA, Leopoldo GO, Duarte JAH, et al. Percepções dos estudantes de Medicina da Ufop sobre sua qualidade de vida. Rev Bras Educ Med. 2014;38(4):435-43 [acesso em 4 dez 2019]. Disponível em: https://www.scielo.br/scielo.php?script=sci_ arttext\&pid=S0100-55022014000400004\&lng=en\&nrm=iso.

29. Gan GG, Hue YL. Anxiety, depression and quality of life of medical students in Malaysia. Med J Malaysia. 2019;74(1):57-61.

30. Cecil J, McHale C, Hart J, Laidlaw A. Behaviour and burnout in medical students. Med Educ Online. 2014;19:25209. doi: 10.3402/ meo.v19.25209.

31. Maia DAC, Maciel RH, Vasconcelos JA, Vasconcelos LA, Filho JOV. Síndrome de Burnout em Estudantes de Medicina: Relação com a Prática de Atividade Física. Cade ESP [on line]. 2012; 6(2):50-9. [capturado 4 dez 2019]. Disponível em: https://cadernos.esp.ce.gov. br/index.php/cadernos/article/view/57/62

32. Ortega ME, Ortiz GR, Martínez AJ. Burnout en estudiantes de pregrado de medicina y su relación con variables de personalidad. Ter Psicol. 2014;32(3):235-42. doi: 10.4067/S0718-48082014000300006.

33. Colby L, Mareka M, Pillay S, Sallie F, van Staden C, du Plessis ED, et al. The association between the levels of burnout and quality of life among fourth-year medical students at the University of the Free State. S Afr J Psychiatr. 2018;24:1101. doi: 10.4102/sajpsychiatry. v24i0.1101.

34. Barker R, Cornwell J, Gishen F. Introducing compassion into the education of health care professionals; can Schwartz Rounds help? J Compassionate Health Care. 2016;3(3) [acesso em 4 abr 2019]. Disponível em: https://discovery.ucl.ac.uk/id/eprint/10060031/.

35. Aherne D, Farrant K, Hickey L, McGrath L, McGrath D. Mindfulness based stress reduction for medical students: optimising student satisfaction and engagement. BMC Med Educ. 2016;16 (209) [acesso em 4 abr 2019]. Disponível em: https://bmcmededuc.biomedcentral. com/articles/10.1186/s12909-016-0728-8.

\section{CONTRIBUIÇÃO DOS AUTORES}

Priscila Gadelha Cazolari participou da conceituação, da curadoria de dados, da análise formal, da investigação, da aquisição de financiamento e da escrita. Matheus de Sousa Cavalcante participou da curadoria de dados, da análise formal, da investigação e da escrita. Marcelo Marcos Piva Demarzo participou da conceituação, da curadoria de dados, da análise formal, da metodologia, da supervisão e da escrita. Frederico Molina Cohrs participou da curadoria de dados, da análise formal, da metodologia, do processamento dos dados com o software, da validação e da escrita. Adriana Sanudo participou da curadoria de dados, da análise formal, do processamento dos dados com o software, da validação e da escrita. Mariana Cabral Schveitzer participou da conceituação, da curadoria de dados, da análise formal, da investigação, da metodologia e da escrita.

\section{CONFLITO DE INTERESSES}

Os autores declaram não haver conflito de interesses neste estudo.

\section{ENDEREÇO PARA CORRESPONDÊNCIA}

Mariana Cabral Schveitzer. Rua Botucatu, 740, $4^{\circ}$ andar, Vila Clementino, São Paulo, SP, Brasil. CEP: 04023-900.

E-mail: mariana.cabral@unifesp.br 